\title{
Unfolding of differential energy spectra in the MAGIC experiment
}

\author{
J. Albert ${ }^{\mathrm{a}}$, E. Aliu ${ }^{\mathrm{b}}, \mathrm{H}$. Anderhub ${ }^{\mathrm{c}}$, P. Antoranz ${ }^{\mathrm{d}}$, \\ A. Armada ${ }^{\text {b }}$, M. Asensio ${ }^{d}$, C. Baixeras ${ }^{\text {e }}$, J. A. Barrio ${ }^{\mathrm{d}}$, \\ H. Bartko ${ }^{f}$, D. Bastieri ${ }^{\mathrm{g}}$, J. Becker ${ }^{\mathrm{h}}$, W. Bednarek ${ }^{\mathrm{i}}$, \\ K. Berger ${ }^{a}$, C. Bigongiari ${ }^{\mathrm{g}}$, A. Biland ${ }^{\mathrm{c}}$, R. K. Bock ${ }^{\mathrm{f}, \mathrm{g}}$, \\ P. Bordas ${ }^{j}$, V. Bosch-Ramon ${ }^{j}$, T. Bretz $^{\text {a }}$, I. Britvitch ${ }^{\mathrm{c}}$, \\ M. Camara ${ }^{\mathrm{d}}$, E. Carmona ${ }^{\mathrm{f}}$, A. Chilingarian ${ }^{\mathrm{k}}$, S. Ciprini ${ }^{\ell}$, \\ J. A. Coarasa ${ }^{f}$, S. Commichau ${ }^{c}$, J. L. Contreras ${ }^{d}$, J. Cortina ${ }^{b}$, \\ M. T. Costado ${ }^{\mathrm{m}, \mathrm{v}}$, V. Curtef $^{\mathrm{h}}$, V. Danielyan ${ }^{\mathrm{k}}$, F. Dazzi ${ }^{\mathrm{g}}$, \\ A. De Angelis ${ }^{n}$, C. Delgado ${ }^{\mathrm{m}}$, R. de los Reyes ${ }^{\mathrm{d}}$, B. De Lotto ${ }^{\mathrm{n}}$, \\ E. Domingo-Santamaría ${ }^{b}$, D. Dorner ${ }^{a}$, M. Doro ${ }^{\mathrm{g}}$, M. Errando $^{\mathrm{b}}$, \\ M. Fagiolini $^{\circ}$, D. Ferenc ${ }^{\mathrm{p}}$, E. Fernández ${ }^{\mathrm{b}}$, R. Firpo $^{\mathrm{b}}$, J. Flix ${ }^{\mathrm{b}}$, \\ M. V. Fonseca ${ }^{d}$, L. Font ${ }^{\mathrm{e}}$, M. Fuchs ${ }^{\mathrm{f}}$, N. Galante ${ }^{\mathrm{f}}$, \\ R. J. García-López ${ }^{\mathrm{m}, \mathrm{v}}$, M. Garczarczyk ${ }^{\mathrm{f}}$, M. Gaug ${ }^{\mathrm{m}}$, \\ M. Giller ${ }^{\mathrm{i}}$, F. Goebel ${ }^{\mathrm{f}}$, D. Hakobyan ${ }^{\mathrm{k}}$, M. Hayashida ${ }^{\mathrm{f}}$, \\ T. Hengstebeck ${ }^{\mathrm{q}}$, A. Herrero ${ }^{\mathrm{m}, \mathrm{v}}$, D. Höhne ${ }^{\mathrm{a}}$, J. Hose ${ }^{\mathrm{f}}$, \\ C. C. Hsu ${ }^{\text {f }}$, P. Jacon ${ }^{\mathrm{i}}$, T. Jogler ${ }^{\mathrm{f}}$, R. Kosyra ${ }^{\mathrm{f}}$, D. Kranich ${ }^{\mathrm{c}}$, \\ R. Kritzer ${ }^{\mathrm{a}}$, A. Laille ${ }^{\mathrm{p}}$, E. Lindfors ${ }^{\ell}$, S. Lombardi ${ }^{\mathrm{g}}$, F. Longo $^{\mathrm{n}}$, \\ J. López $^{\mathrm{b}}$, M. López ${ }^{\mathrm{d}}$, E. Lorenz ${ }^{\mathrm{c}, \mathrm{f}}$, P. Majumdar ${ }^{\mathrm{f}}$, \\ G. Maneva ${ }^{\mathrm{r}}, \mathrm{K}_{\text {. Mannheim }}{ }^{\mathrm{a}}$, O. Mansutti ${ }^{\mathrm{n}}$, M. Mariotti $^{\mathrm{g}}$,

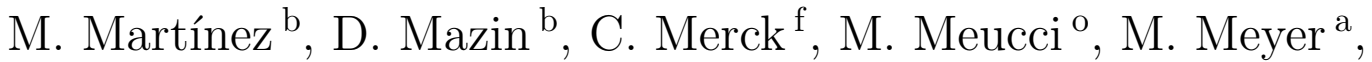 \\ J. M. Miranda ${ }^{\text {d }}$, R. Mirzoyan ${ }^{\text {f }}$, S. Mizobuchi ${ }^{\mathrm{f}}$, A. Moralejo ${ }^{\mathrm{b}}$, \\ D. Nieto ${ }^{\mathrm{d}}$, K. Nilsson ${ }^{\ell}$, J. Ninkovic ${ }^{\mathrm{f}}$, E. Oña-Wilhelmi ${ }^{\mathrm{b}}$, \\ N. Otte ${ }^{\text {f,q }}$, I. Oya ${ }^{\mathrm{d}}$, M. Panniello ${ }^{\mathrm{m}, \mathrm{w}}$, R. Paoletti ${ }^{\mathrm{o}}$, \\ J. M. Paredes ${ }^{j}$, M. Pasanen ${ }^{\ell}$, D. Pascoli ${ }^{g}$, F. Pauss ${ }^{c}$, \\ R. Pegna ${ }^{o}$, M. Persic ${ }^{n, s}$, L. Peruzzo $^{g}$, A. Piccioli ${ }^{\circ}$, \\ N. Puchades ${ }^{b}$, E. Prandini ${ }^{\mathrm{g}}$, A. Raymers ${ }^{\mathrm{k}}$, W. Rhode ${ }^{\mathrm{h}}$, \\ M. Ribó ${ }^{j}$, J. Rico ${ }^{b}$, M. Rissi $^{c}$, A. Robert $^{\mathrm{e}}$, S. Rügamer ${ }^{\mathrm{a}}$, \\ A. Saggion ${ }^{g}$, T. Saito ${ }^{f}$, A. Sánchez ${ }^{e}$, P. Sartori ${ }^{g}$, V. Scalzotto ${ }^{g}$, \\ V. Scapin ${ }^{n}$, R. Schmitt ${ }^{\text {a }}$, T. Schweizer ${ }^{\mathrm{f}}$, M. Shayduk ${ }^{\mathrm{q}, \mathrm{f}}$, \\ K. Shinozaki ${ }^{\mathrm{f}}$, S. N. Shore ${ }^{\mathrm{t}}$, N. Sidro ${ }^{\mathrm{b}}$, A. Sillanpää ${ }^{\ell}$,
}




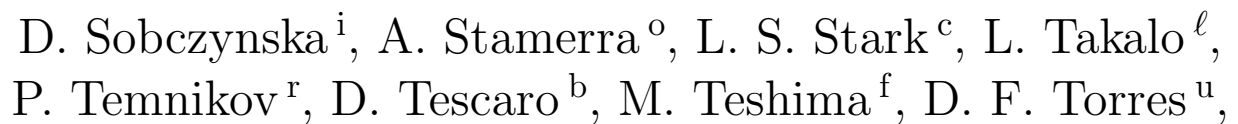

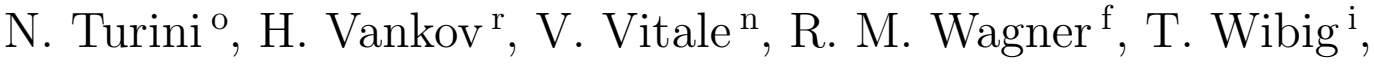
W. Wittek ${ }^{\mathrm{f}, *}$, F. Zandanel $^{\mathrm{g}}$, R. Zanin ${ }^{\mathrm{b}}$, J. Zapatero ${ }^{\mathrm{e}}$

${ }^{a}$ Universität Würzburg, D-97074 Würzburg, Germany

${ }^{\mathrm{b}}$ Institut de Física d'Altes Energies, Edifici Cn., E-08193 Bellaterra (Barcelona), Spain

${ }^{\mathrm{c}}$ ETH Zurich, CH-8093 Switzerland

${ }^{\mathrm{d}}$ Universidad Complutense, E-28040 Madrid, Spain

${ }^{\mathrm{e}}$ Universitat Autònoma de Barcelona, E-08193 Bellaterra, Spain

${ }^{\mathrm{f}}$ Max-Planck-Institut für Physik, D-80805 München, Germany

g Università di Padova and INFN, I-35131 Padova, Italy

${ }^{\mathrm{h}}$ Universität Dortmund, D-44227 Dortmund, Germany

i University of Eódź, PL-90236 Lodz, Poland

j Universitat de Barcelona, E-08028 Barcelona, Spain

${ }^{\mathrm{k}}$ Yerevan Physics Institute, AM-375036 Yerevan, Armenia

${ }^{\ell}$ Tuorla Observatory, FI-21500 Piikkiö, Finland

${ }^{\mathrm{m}}$ Inst. de Astrofisica de Canarias, E-38200, La Laguna, Tenerife, Spain

${ }^{\mathrm{n}}$ Università di Udine, and INFN Trieste, I-33100 Udine, Italy

${ }^{\circ}$ Università di Siena, and INFN Pisa, I-53100 Siena, Italy

p University of California, Davis, CA-95616-8677, USA

${ }^{\mathrm{q}}$ Humboldt-Universität zu Berlin, D-12489 Berlin, Germany

${ }^{\mathrm{r}}$ Institute for Nuclear Research and Nuclear Energy, BG-1784 Sofia, Bulgaria

${ }^{\mathrm{s}}$ INAF/Osservatorio Astronomico and INFN Trieste, I-34131 Trieste, Italy

${ }^{\mathrm{t}}$ Università di Pisa, and INFN Pisa, I-56126 Pisa, Italy

u ICREA 8 Institut de Ciències de l'Espai (CSIC-IEEC), E-08193 Bellaterra, Spain

${ }^{\vee}$ Depto. de Astrofisica, Universidad, E-38206, La Laguna, Tenerife, Spain

${ }^{\mathrm{w}}$ deceased

\begin{abstract}
The paper describes the different methods, used in the MAGIC experiment, to unfold experimental energy distributions of cosmic ray particles ( $\gamma$-rays). Questions and problems related to the unfolding are discussed. Various procedures are proposed which can help to make the unfolding robust and reliable. The different methods and procedures are implemented in the MAGIC software and are used in most of the analyses.
\end{abstract}


Key words:

\section{Introduction}

In an Imaging-Air-Cherenkov-Telescope (IACT) experiment like MAGIC [1] the energy $E$ of the cosmic ray particle ( $\gamma$-ray) is not exactly known. It has to be estimated, the energy resolution being in the order of 20 to $40 \%$. As a consequence, the experimentally measured energy spectrum is biased. The procedure to correct for the effects due to the finite energy resolution is called unfolding.

While in high-energy-physics experiments unfolding is a widely used technique, this is not the case in present day's IACT experiments. This paper deals with the unfolding procedure, which is applied as a standard tool in the MAGIC experiment. The different unfolding methods are explained in detail. Emphasis is put on the discussion of questions and problems related to the application of the unfolding to real data. It is not the aim of the paper to give a complete derivation of all formulas. For this the reader is referred to the publications [2] to [18]. An excellent review of unfolding methods is given in [13]. The present paper makes use of many ideas discussed in that paper.

Although here only differential energy spectra are considered, the procedures are equally well applicable to distributions of other quantities, including distributions in more than one dimension [19].

The layout of the paper is as follows. In Section 2 the notation is defined and the motivation for the unfolding procedure is given. The different unfolding methods, which means the different ways of regularization, are presented in Section 3. The so-called Forward Unfolding, which represents an implicit unfolding under the assumption of a certain parametrization of the unfolding result $S$, is explained in Section 4. In Section 5 two quantities are introduced, which are useful for an optimal choice of the regularization strength. The criteria for this choice are collected in Section 6. Section 7 discusses various technical aspects which are important in the application of the unfolding procedure to real data. Two particular technical procedures, which ensure an unbiased and robust unfolding, are presented in Sections 8 and 9. Some unfolding results, obtained by applying the unfolding procedure to data taken in the MAGIC experiment, are discussed in Section 10. Finally the pros and contras of the method of Correction Factors, which is an alternative way of

\footnotetext{
* Corresponding author.

Email address: wittek@mppmu.mpg.de (W. Wittek).
} 
correcting data for effects due to the finite experimental resolution, are listed in Section 11. A summary is given in Section 12.

\section{The aim of the unfolding procedure}

In this Section the notations are defined and the motivation for the unfolding procedure is explained.

\subsection{Notation}

The true and measured (estimated) values of the energy of the cosmic ray particle are denoted by $E_{\text {true }}$ and $E_{\text {est }}$ respectively. The data are assumed to be binned in histograms, and certain binnings are chosen independently for the distributions in $E_{\text {true }}$ and $E_{\text {est }}$. Furthermore, the following definitions are introduced:

$$
\begin{array}{cll}
Y_{i} & \text { number of events in bin } i \text { of } E_{e s t} & (i=1, \ldots n a) \\
K_{i, k} & \text { covariance matrix } K \text { of } Y & (i=1, \ldots n a ; k=1, \ldots n a) \\
S_{j} & \text { number of events in bin } j \text { of } E_{\text {true }} & (j=1, \ldots n b) \\
T_{j, l} & \text { covariance matrix } T \text { of } S & (j=1, \ldots n b ; l=1, \ldots n b) \\
M_{i j} & \text { migration matrix } M & (i=1, \ldots n a ; j=1, \ldots n b) \\
G_{i k} & \text { Gram's matrix } G=M \cdot M^{T} & (i=1, \ldots n a ; k=1, \ldots n a)
\end{array}
$$

with

$$
\sum_{i=1}^{n a} M_{i j}=1 \quad \text { for all } j
$$

In the following it is assumed that the rank $n r$ of $G$ is equal to (and not less than) the minimum of $n a$ and $n b$, where $n a$ and $n b$ are the number of bins in $E_{\text {est }}$ and $E_{\text {true }}$ respectively, which are used in the unfolding. This can always be achieved by a proper choice of the binnings in $E_{\text {true }}$ and $E_{\text {est }}$.

The migration matrix contains the most likely fraction of events moving from a bin $j$ in $E_{\text {true }}$ into a bin $i$ of $E_{\text {est }}$, due to the finite experimental energy 
resolution:

$$
\begin{aligned}
Y_{i} & =\sum_{j=1}^{n b} M_{i j} \cdot S_{j} \quad(i=1, \ldots n a) \\
\text { or in matrix notation } \quad Y & =M \cdot S
\end{aligned}
$$

The migration matrix $M$ is obtained from Monte Carlo (MC) simulations, in which the development of the air shower (induced by the cosmic ray particle in the atmosphere), the emission of Cherenkov light in the air shower, the geometrical, optical and electronic properties of the telescope and the experimental procedures in the data analysis (shower reconstruction, $\gamma /$ hadron separation, energy estimation, selections, cuts) are simulated [20]. $M$ is computed from a 2-dimensional plot of the number of reconstructed MC events in the $E_{\text {est }}-E_{\text {true }}$ plane, which was produced under the same conditions (selections, cuts) as the experimental distribution $Y$. It is the aim of the unfolding procedure to determine the true distribution $S$, given $Y$ and $M$.

It should be noted that $M$ only describes the migration of events. It does not describe losses of events, which will occur due to the finite acceptance of the detector, due to the trigger conditions and due to additional selections and cuts. In an IACT experiment these losses are also determined by Monte Carlo simulations, and the corresponding correction factor is the effective collection area $A_{\text {eff }}\left(E_{\text {true }}\right)$. $A_{\text {eff }}\left(E_{\text {true }}\right)$ has to be computed again under the same conditions as the experimental distribution $Y$. Apart from minor effects (see Section 8 ), this correction can be performed quite independently of the unfolding.

The unfolding can be understood as a reshuffling of events from the bins of $E_{\text {est }}$ into the bins of $E_{\text {true }}$. In this procedure the numerical values of the bin edges, both for $E_{\text {true }}$ and $E_{\text {est }}$, are completely irrelevant. $E_{\text {true }}$ and $E_{\text {est }}$ may be even two different physical quantities, with completely different ranges of values and different units, like $E_{\text {true }}=$ (the true energy of the cosmic ray particle) and $E_{\text {est }}=$ (the total number of Cherenkov photons measured for the shower) [21]. Of course, unfolding makes only sense if $E_{\text {true }}$ and $E_{\text {est }}$ are sufficiently strongly correlated, otherwise the distribution of $E_{\text {true }}$ cannot be inferred from a distribution of $E_{\text {est }}$. This is in contrast to the method of Correction Factors (see Section 11), where $E_{\text {est }}$ has to be a good estimate of $E_{\text {true }}$ in any case.

Because the binnings in $E_{\text {true }}$ and $E_{\text {est }}$ can be chosen independently and to a certain degree arbitrarily (see Section 7 ), the migration matrix $M$ is in general not square. For this reason eq. (2) can in general not be inverted to obtain $S$ as $M^{-1} \cdot Y$. 
As the unfolding is equivalent to a reshuffling of events from the bins of the measured distribution into the bins of the true distribution the unfolding is not restricted to 1-dimensional distributions but can in the same way be applied to multi-dimensional distributions. The information necessary for the unfolding procedure is completely contained in the corresponding migration matrix, which in the case of multi-dimensional distributions describes the migration of events from the bins of the true multi-dimensional distribution into the bins of the measured multi-dimensional distribution. The dimensions of the measured and true distributions may also be different. An example for an unfolding in 2 dimensions is given in [19].

\subsection{The direct solution of $Y=M \cdot S$}

Very generally, the solution $S$ of the system of linear equations (2) can be obtained by minimizing the Least-Squares expression

$$
\chi_{0}^{2}=(Y-M \cdot S)^{T} \cdot K^{-1} \cdot(Y-M \cdot S)
$$

where the $n b$ components of $S$ are the free parameters. Minimizing $\chi_{0}^{2}$ will yield solutions for $S$ which, after folding with $M$, are best compatible with the measurement $Y$.

Two cases have to be distinguished:

- The underconstrained case $n r=n a \leq n b$.

Because $n r=n a$ the $n a \times n a$ matrix $G$ can be inverted and a particular solution $S_{0}$ can be written as

$$
S^{0}=M^{T} \cdot C \quad \text { with } \quad C=G^{-1} \cdot Y
$$

If $n a<n b$, the solutions $S=S_{0}+S_{T}$ form a space of $(n b-n a)$ dimensions with $M \cdot S_{T}=0$. For $n a=n b$ eq.(4) reduces to

$$
S^{0}=M^{T} \cdot G^{-1} \cdot Y=M^{-1} \cdot Y
$$

and $S^{0}$ is the only solution. In both cases $M \cdot S=M \cdot S^{0}=Y$, implying $\chi_{0}^{2}=$ 0 . The solutions are independent of the covariance matrix $K$. Moreover, because of (1) the total number of events is not changed:

$$
\sum_{i} Y_{i}=\sum_{i} \sum_{j} M_{i j} \cdot S_{j}^{0}=\sum_{j} S_{j}^{0}
$$

- The overconstrained case $n a>n b=n r$.

Minimizing $\chi_{0}^{2}$ by varying $S$ yields 


$$
S^{L S Q}=H^{-1} M^{T} K^{-1} \cdot Y
$$

where $H$ is the $n b \times n b$ matrix

$$
H=M^{T} K^{-1} M
$$

The solution $S^{L S Q}$ now depends on $K$. The minimum value of $\chi_{0}^{2}$ becomes

$$
\chi_{0}^{2}=\left(Y-M \cdot S^{L S Q}\right)^{T} \cdot K^{-1} \cdot\left(Y-M \cdot S^{L S Q}\right)
$$

Expression (7) is also valid for $n r=n a=n b$, in which case it reduces to $S^{L S Q}=M^{-1} \cdot Y$.

It can be shown that the direct solutions (4) and (7) may lead to large errors of $S_{j}$, reflected in large absolute values of the elements of the error matrix $T$ of $S$. This behaviour can be traced back to small eigenvalues of the matrix $G$ and $H$ respectively [13].

\section{Unfolding with Regularization - The different Unfolding Meth- ods}

In order to reduce the large errors of $S$, a procedure called regularization is applied. By the regularization additional constraints are imposed on $S$, by which some information in the measurements $Y_{i}$ is discarded. Regularization can be viewed as a smearing of the unfolded distribution with some finite resolution, which reduces the correlations between the $S_{i}$ of adjacent bins at the expense that $S_{i}$ is no longer an unbiased estimate of the true distribution [17].

The bias increases with increasing regularization strength. Nevertheless, it turns out that with properly tuned regularization (see Section 6) solutions can be obtained which are much closer to the true distribution than the direct solutions (4) or (7).

It is evident that regularization is particularly important in the underconstrained case. However, also in the overconstrained case regularization makes sense: even if the system of equations (2) is formally overconstrained ( $n a>$ $n b$ ), it may be effectively underconstrained. This happens for example if some of the measurements $Y_{i}$ have much larger errors than the other $Y_{i}$.

In the following, three different ways of regularization are described [13]. 


\subsection{Adding a regularization term in the expression for $\chi_{0}^{2}$}

In some unfolding methods regularization is performed by adding a regularization term $\operatorname{Reg}(S)$ in the expression for $\chi_{0}^{2}$ (eq.3)

$$
\chi^{2}=\frac{w}{2} \cdot \chi_{0}^{2}+\operatorname{Reg}(S)
$$

$w$, also called regularization parameter, is a weight which allows to steer the regularization strength: large values of $w$ correspond to weak regularization, small values to strong regularization.

\section{- Tikhonov's method}

In Tikhonov's method [4] the regularization term is defined as

$$
\operatorname{Reg}(S)=\sum_{j=1}^{n b}\left(\frac{d^{2} S}{d x^{2}}\right)_{j}^{2}
$$

For the second derivative $\left(\frac{d^{2} S}{d x^{2}}\right)_{j}$ of $S$ in bin $j$ different approximations may be used. The expression used in the MAGIC software [22] is

$$
\left(\frac{d^{2} S}{d x^{2}}\right)_{j}=2.0 \cdot\left(\frac{S_{j+1}-S_{j}}{S_{j+1}+S_{j}}-\frac{S_{j}-S_{j-1}}{S_{j}+S_{j-1}}\right)
$$

This is actually an approximation for the bin-to-bin variation of $\Delta S / S$. In $[7]\left(\frac{d^{2} S}{d x^{2}}\right)_{j}$ is calculated from a spline representation of $S$.

For a given value of $w$ and after specifying $\operatorname{Reg}(S)$, expression (10) can be minimized numerically by varying the components of $S$. The minimization also provides the error matrix $T$ of $S$. The regularization matrix $R$, defined in (24), can be calculated numerically by performing minimizations with modified values of $Y_{i}$.

\section{- Schmelling's method}

In this method, which is discussed in great detail in $[14,17]$, the regularization term is set equal to the "cross entropy"

$$
\operatorname{Reg}(S)=\sum_{j=1}^{n b} p_{j} \cdot \ln \frac{p_{j}}{\epsilon_{j}}
$$

$p_{j}$ is the normalized distribution $S$ 


$$
p_{j}=\frac{S_{j}}{\sum_{k=1}^{n b} S_{k}} \quad \sum_{j=1}^{n b} p_{j}=1
$$

and $\epsilon$ is a normalized prior distribution, which describes a prior knowledge about $S$. The cross entropy $\operatorname{Reg}(S)$ quantifies by how much $p$ deviates from $\epsilon$. Finding $S$ by minimizing the cross entropy $\operatorname{Reg}(S)$ simultaneously with the least squares expression $\chi_{0}^{2}$ is called the method of "Reduced Cross Entropy".

With $\operatorname{Reg}(S)$ from (13), expression (10) can now be minimized to obtain the unfolded distribution $S$. Note that all components of $S$ and of the prior distribution are required to be $>0$, because otherwise $\operatorname{Reg}(S)$ in (13) cannot be defined. The expressions for the error matrix $T$ of $S$ and for the regularization matrix $R$ are given in $[14,17]$.

In the MAGIC software, the condition $\sum_{i} Y_{i}=\sum_{j} S_{j}$ is used as an additional constraint, when minimizing $\chi^{2}$. By this one degree of freedom is gained.

\subsection{Spectral Window method}

In some unfolding methods regularization is performed by suppresing small eigenvalues $\lambda_{l}$ of $G$ by a factor $f\left(\lambda_{l}\right)$ [11]. By the suppression factor $f\left(\lambda_{l}\right)$ the matrix $G$ and its inverse are modified. In terms of the eigenvectors $g_{l}$ of $G$ they read

$$
\begin{aligned}
\widetilde{G} & =\sum_{l}^{n r} f\left(\lambda_{l}\right) \cdot \lambda_{l} \cdot g_{l} g_{l}^{T} \\
\widetilde{G^{-1}} & =\sum_{l}^{n r} \frac{f\left(\lambda_{l}\right)}{\lambda_{l}} \cdot g_{l} g_{l}^{T}
\end{aligned}
$$

where the sums extend over all eigenvalues $\lambda_{l}$ which are different from zero. Like $G$ and $G^{-1}, \widetilde{G}$ and $\widetilde{G^{-1}}$ are $n a \times n a$ matrices. Without suppression, $f\left(\lambda_{l}\right)=1, \widetilde{G^{-1}}$ is equal to $G^{-1}$ in the underconstrained case $n r=n a \leq n b$. In the overconstrained case, $n a>n b=n r, G^{-1}$ is undefined but $\widetilde{G^{-1}}$ can be calculated.

A similar factor $f\left(\kappa_{l}\right)$ can be defined to suppress small eigenvalues $\kappa_{l}$ of $H$ (eq. $8)$. There is considerable freedom as to the choice of the values or expressions for $f\left(\lambda_{l}\right)$ and $f\left(\kappa_{l}\right)$. One may introduce a parameter $i$ such that in the limit $i \rightarrow \infty$ the suppression factors $f\left(\lambda_{l}, i\right)$ and $f\left(\kappa_{l}, i\right)$ tend to 1 . $i$ has a similar meaning as the weight $w$ in eq.(10): it determines the regularization strength and for $i \rightarrow \infty$ the solutions tend to the direct solutions (4) and (7) respectively. 
The expressions for the error matrix $T$ of $S$ and for the regularization matrix $R$ are given in [13].

\subsection{Regularization by iteration}

Another way of unfolding is to calculate a solution $S$ iteratively $[3,9,11,12]$. The regularization is done by stopping the iteration at some point. In this case the number of iterations $i$ plays a similar role as the weight $w$ in (10). In the limit of an infinite number of iterations, which is equivalent to a very large weight $w$, the solution tends to the direct solution (4) or (7) respectively.

- $\mathbf{n r}=\mathbf{n a} \leq \mathbf{n b}$

A simple iteration scheme [13] for solving $Y=M \cdot S$ with $G^{-1} \cdot Y=C$ is

$$
C^{i+1}=C^{i}-\tau \cdot\left(G C^{i}-Y\right)
$$

where $i$ is the iteration number and $\tau$ is a relaxation parameter. The latter should be chosen in the range $0<\tau<2 / \lambda_{\max }$, where $\lambda_{\max }$ is the largest eigenvalue of $G$. Eq.(17) leads to

$$
C^{i}=(1-\tau G)^{i} \cdot C^{0}+\tau \cdot \sum_{j=0}^{i-1}(1-\tau G)^{j} \cdot Y
$$

where $C^{0}$ is the starting value of $C$. The unfolded distribution is obtained as $S^{i}=M^{T} \cdot C^{i}$.

In terms of the suppression factor $f\left(\lambda_{l}, i\right)$ this regularization can be expressed as [13]

$$
f\left(\lambda_{l}, i\right)=\left[1-\left(1-\tau \lambda_{l}\right)^{i}\right]
$$

if $C^{0}$ is set to zero, and

$$
f\left(\lambda_{l}, i\right)=\left[1-\left(1-\tau \lambda_{l}\right)^{i}+\left(1-\tau \lambda_{l}\right)^{i} \lambda_{l}\right]
$$

if $C^{0}$ is chosen to be equal to $Y$. The solutions $S^{i}$ tends to the direct solution $S^{0}=M^{T} \cdot C=M^{T} \cdot G^{-1} \cdot Y$ (eq. (4)).

This procedure can also be applied in the overconstrained case, $n a>$ $n b=n r$. In this case $G^{-1}$ is undefined and it has to be replaced by $\widetilde{G^{-1}}$ (eq.16). The solutions $S^{i}$ tend to $S=M^{T} \cdot \widetilde{G^{-1}} \cdot Y$, with $f\left(\lambda_{l}\right)=1$. 
- $\mathbf{n a}>\mathbf{n b}=\mathbf{n r}$

In a similar way one may define suppression factors $f\left(\kappa_{l}, i\right)$ for the eigenvalues of the matrix $H$. In this case the solution $S$ tends to the solution $S^{L S Q}$ (eq. (7)).

In the last 2 methods, Spectral Window method and Regularization by iteration, $\chi_{0}^{2}$ doesn't appear explicitly. However, its value can be calculated and it is taken into account when determining the optimum regularization strength (see Section 6).

Once the the unfolded distribution $S_{k}$ is determined, by any of the methods described in this Section, the differential energy spectrum $\Phi_{k}$ of $\gamma$-rays is calculated using eq. (22). $\Phi_{k}$ has the meaning of the average differential $\gamma$-ray flux in the $k$-th bin of $E_{\text {true }}$ (eq.(23)).

\section{Forward Unfolding}

An implicit unfolding can be done by representing $S$ as a parametric function $S_{k}(q)=f\left(E_{k} ; q\right)$ with parameters $q=\left(q_{1}, q_{2}, \ldots q_{n q}\right)$ and minimizing $\chi_{0}^{2}$ in $(3)$

$$
\chi_{0}^{2}=\sum_{i, j=1}^{n a}\left(Y_{i}-\sum_{k=1}^{n b} M_{i k} \cdot S_{k}(q)\right) \cdot\left(K^{-1}\right)_{i j} \cdot\left(Y_{j}-\sum_{l=1}^{n b} M_{j l} \cdot S_{l}(q)\right)
$$

with respect to the parameters $q$. The number of measurements is equal to $n a$, the number of unknowns $n q$. Thus, the problem is overconstrained if $n q<n a$, independent of the value of $n b$. One degree of freedom is gained if the total number of events is required to stay constant: $\sum_{j} S_{j}=\sum_{i} Y_{i}$.

In many cases the minimization of $\chi_{0}^{2}$ can be performed analytically, by solving $\frac{\partial \chi_{0}^{2}}{\partial q}=0$, similarly to the procedure described in Section 2.2 .

The parametrization of $S$ can be written in the form

$$
S_{k}(q)=\Phi_{k}(q) \cdot T_{\text {eff }} \cdot \Delta\left(E_{\text {true }}^{k}\right) \cdot A_{\text {eff }}^{k} \cdot A_{\text {addcut }}^{k} \cdot A_{\text {absorption }}^{k}
$$

with

$$
\Phi_{k}(q)=\frac{\int_{\Delta\left(E_{\text {true }}^{k}\right)} \Phi\left(E_{\text {true }}, q\right) \cdot d E_{\text {true }}}{\Delta\left(E_{\text {true }}^{k}\right)}
$$


Here $\Phi\left(E_{\text {true }}, q\right)$ is the assumed parametrization of the differential energy spectrum of $\gamma$-rays, $T_{\text {eff } f}$ is the effective observation time, $\Delta\left(E_{\text {true }}^{k}\right)$ denotes the $k$-th bin in $E_{\text {true }}$ or its width, $A_{e f f}^{k}$ is the effectice collection area, and $A_{\text {addcut }}^{k}$ is the reduction factor due to an additional cut (for example in $E_{\text {est }}$, see Section 7.3). A further correction $A_{\text {absorption }}^{k}$ can be introduced, if $\Phi\left(E_{\text {true }}, q\right)$ is supposed to represent the differential energy spectrum of $\gamma$-rays before absorption, either at the $\gamma$-source or by interaction with the extragalactic photon background. $A_{\text {addcut }}^{k}$ can be determined from MC simulations, whereas $A_{\text {absorption }}^{k}$ can be calculated in models about the extragalactic photon background [23].

Parametrizing $S$ as an analytic function of $E_{\text {true }}$, with some free parameters $q$, can be understood as a kind of regularization, because it forces the solution $S$ and its derivatives to be continuous, leading to a suppression of the noise component of $S$.

The Forward Unfolding does not provide an unfolded distribution $S$. It provides those parameter values $q$ for an assumed parametrization of $\Phi\left(E_{\text {true }}\right)$, which minimize $\chi_{0}^{2}$ in (21). Of course, $S$ can then be calculated from $\Phi\left(E_{\text {true }}\right)$ via (22).

Under the assumption of a certain parametrization of $\Phi\left(E_{\text {true }}\right)$, the Forward Unfolding is a very robust method of determining the best parameter values $q$. Moreover, since there is no regularization strength to be adjusted, the uncertainty as to its choice does not exist. Therefore Forward Unfolding represents a powerful and useful check of the unfolding results obtained by any of the methods described in Section 3. In those methods the parametrization is only introduced after the actual unfolding of the measurements $Y$.

\section{Useful quantities in the unfolding}

In this Section two quantities are explained which are useful for judging the quality of the unfolding result: The error matrix (covariance matrix) $T$ of the unfolded distribution $S$ and the regularization matrix $R$.

\subsection{The covariance matrix of the unfolded distribution $S$}

In all unfolding methods the covariance matrix $T$ of $S$ can be determined. The trace of $T, \operatorname{Trace}(T)$, measures the noise component of $S$, as $\operatorname{Trace}(K)$ measures the noise component of $Y$.

In the methods where the solution $S$ is given as $S=D \cdot Y$, like in Schmelling's method or in the Spectral Window method, the covariance matrix $T$ of $S$ is 
obtained by $T=D \cdot K \cdot D^{T}$.

In those methods where the solution is determined by a numerical minimiza-

tion of $\chi^{2}$, like in Tikhonov's method, $T$ is obtained from the shape of $\chi^{2}$ in the region around the minimum.

\subsection{The regularization matrix $R$}

A quantity which describes how the estimates $\sum_{k=1}^{n b} M_{j k} S_{k}$ of $Y_{j}$ couple to the measurements $Y_{i}$ is given by the $n a \times n a$ matrix

$$
R_{i j}=\frac{\partial\left(\sum_{k=1}^{n b} M_{j k} S_{k}\right)}{\partial Y_{i}} \quad \text { or } \quad R=\frac{\partial(M \cdot S)}{\partial Y}
$$

also called regularization matrix [17]. The trace of $R$ can be interpreted as the effective number of measurements used in the unfolding procedure. The number of effectively rejected measurements is then equal to $N_{r e j}=n a-\operatorname{Trace}(R)$.

The maximum value of $\operatorname{Trace}(R)$, which is equal to the rank $n r$ of $G$, is reached with the direct solutions (4) and (7), corresponding to the cases $n r=n a \leq n b$ and $n a>n b=n r$ respectively: For the direct solution $S^{0}$ the number of rejected measurements $N_{r e j}$ is equal to $n a-\operatorname{Trace}(R)=n a-n r=n a-$ $n a=0$, which means that no information is discarded. The measurements $Y$ are completely reproduced by the unfolding : $M \cdot S^{0}=Y$. For the least squares solution $S^{L S Q}$ the number of effectively rejected measurements is $N_{r e j}=$ $n a-\operatorname{Trace}(R)=n a-n r=n a-n b>0$, which means that some information is discarded. The measurements $Y$ are not exactly reproduced by the unfolding : $M \cdot S^{L S Q} \neq Y$. The fact that the system is overconstrained has a similar effect as regularization. In both cases, with increasing regularization strength $\operatorname{Trace}(R)$ is reduced and $N_{\text {rej }}$ is increased.

\section{Selecting the unfolding result}

For a given unfolding method the result $S$ depends on the regularization strength, which is given by the weight $w$ (or by the number of iterations $i$ respectively). In the literature various criteria for choosing the "best" weight are proposed $[8,10,13,16-18]$. Unfortunately, none of them provides a choice which is optimal for all cases. Reasons for this are: The optimum regularization strength in general depends on the shape of the unknown distribution $S$. 
It also depends on the binnings in $E_{\text {true }}$ and $E_{\text {est }}$ and on the prior distribution (if applicable).

The effect of the regularization is illustrated in Fig. 1, where different quantities are plotted as a function of the iteration number $i$. In this example an experimental energy distribution of $\gamma$-rays from the Crab Nebula [24], i.e. the number of excess events in bins of the estimated energy $E_{\text {est }}$, was unfolded. The unfolding was performed for 30 different $i$ in the range $10^{-5}$ to $10^{10}$, using the method of Bertero (eq.(20)). More results from the analysis of these data are given in Section 10. With decreasing $i$, i.e with increasing regularization strength, one observes an increase of $\chi_{0}^{2}$ (eq. 3) and a decrease in the quantities $\operatorname{Trace}(T) / \operatorname{Trace}(K), \operatorname{Trace}(R), \operatorname{Reg}(S)_{\text {Tikhonov }}$ (eq. 11) and $\operatorname{Reg}(S)_{\text {Schmelling }}$ (eq. 13). Very similar behavior is found for the other unfolding methods, discussed in Section 3.

Obviously, an acceptable unfolding result should satisfy the following conditions:

- The $\chi^{2}$-probability, calculated from the value of $\chi_{0}^{2}$ and the number of degrees of freedom in the unfolding procedure, should be acceptable, say $>1 \%$. Otherwise the unfolding result is incompatible with the measured distribution $Y$.

- The noise term Trace $(T)$ of the unfolded distribution $S$ should be comparable to the noise term $\operatorname{Trace}(K)$ of the measurements. The main aim of regularization is a suppression of the large noise term of $S$, which one often obtains if no regularization is applied. A large noise term $\operatorname{Trace}(T)$, as well as large correlation terms of $T$, indicate a too fine binning in $E_{\text {true }}$, leading to small eigenvalues of $G$ or $H$ (see Section 7.1).

- $\operatorname{Trace}(R)$ should not be much lower than its maximum possible value, which is equal to the rank $n r$ of the matrix $G$. Otherwise the solution is too strongly dominated and biased by the regularization.

For determining the "best" regularization strength a compromise has to be found between the above requirements. It has turned out that the criterion $\operatorname{Trace}(T)=\operatorname{Trace}(K)$ in general leads to solutions which satisfy the above conditions reasonably well, provided the problem is not strongly overconstrained. In the latter case, where the unfolding result is better constrained, a solution with $\operatorname{Trace}(T)<\operatorname{Trace}(K)$ is more apropriate. In MAGIC the standard criterion for determining the optimal regularization strength is $\operatorname{Trace}(T)=\operatorname{Trace}(K)$. The full circles in Fig. 1 indicate this choice. However, any other regularization strength can be chosen by hand, if this is suggested by the behaviour of the quantities $\chi_{0}^{2}, \operatorname{Trace}(T), \operatorname{Trace}(R), \operatorname{Reg}(S)_{\text {Tikhonov }}$ 


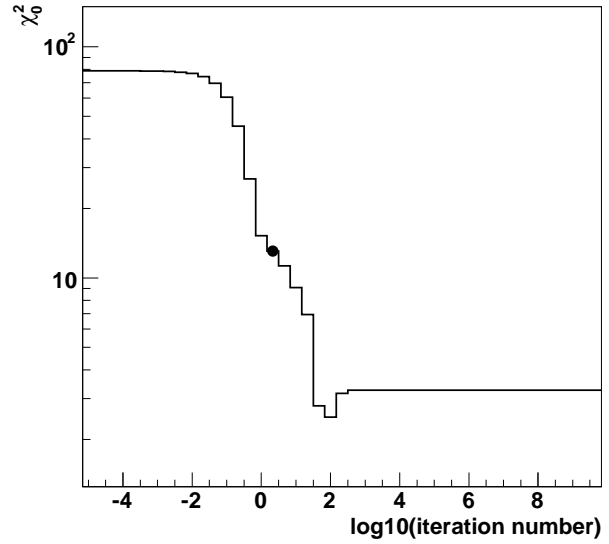

(a) $\chi_{0}^{2}$

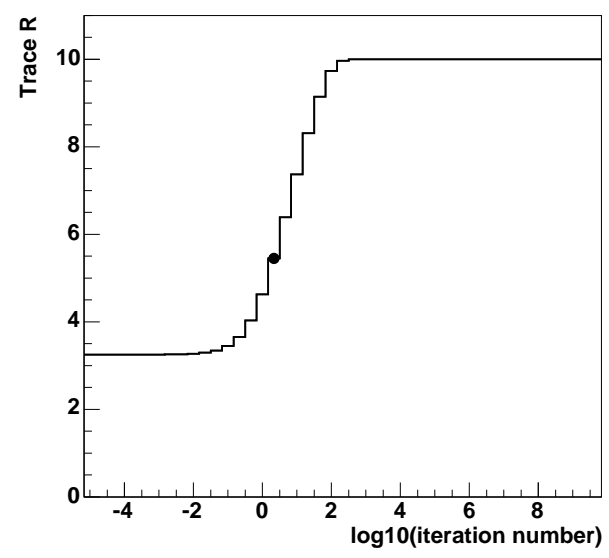

(c) $\operatorname{Trace}(R)$

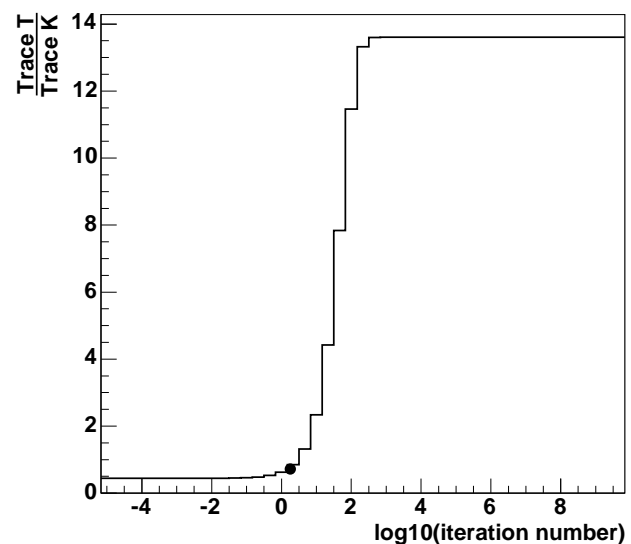

(b) $\operatorname{Trace}(T) / \operatorname{Trace}(K)$

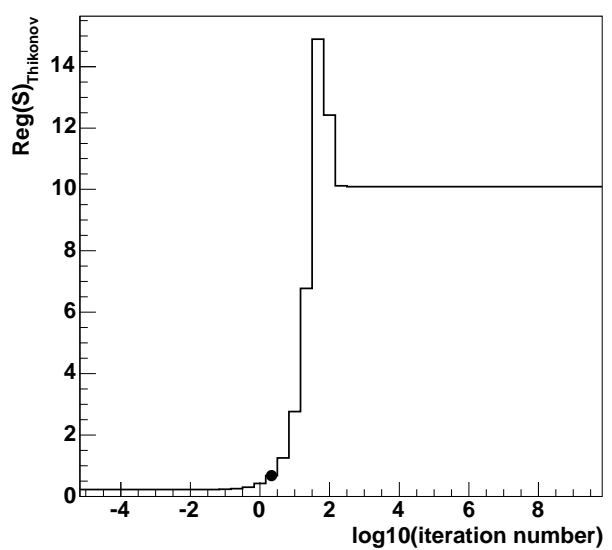

(d) $\operatorname{Reg}(S)_{\text {Tikhonov }}$

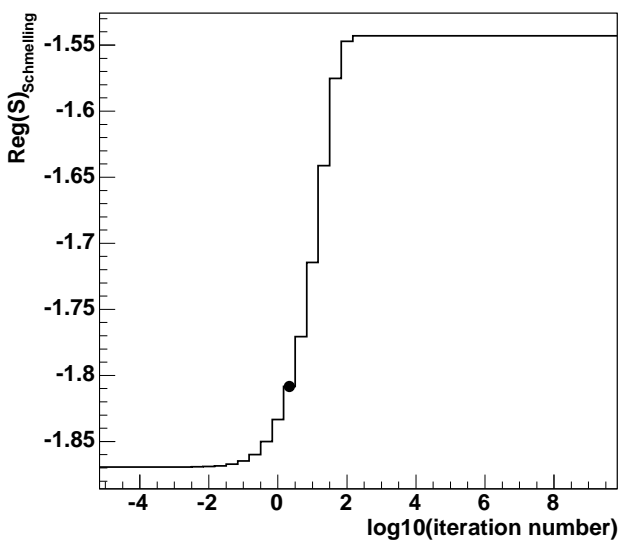

(e) $\operatorname{Reg}(S)_{\text {Schmelling }}$

Fig. 1. Useful quantities for determining the optimum regularization strength, plotted as a function of the iteration number $i$. 
Unfolding with regularization is a procedure which allows freedom in the choice of the regularization method and in the choice of the regularization strength. The above criteria for an acceptable solution strongly restrict this freedom. Nevertheless a certain degree of arbitrariness remains as to which unfolding result should be considered representative and final. In MAGIC a selected unfolding result is considered representative if all other unfolding methods yield results, which are also acceptable and statistically consistent with the selected result. In addition, it is required that also the Forward Unfolding (Section 4$)$, using a reasonable parametrization of $\Phi\left(E_{\text {true }}\right)$, gives a consistent result.

An uncertainty due to the unfolding is determined from the spread of the $S_{j}$, obtained from the different unfolding methods.

\section{$7 \quad$ Further comments on the unfolding}

In the actual application of the unfolding procedure to real data some technical details have to be considered, and they are discussed in this Section.

\subsection{Optimal binnings}

The binning of the experimental distribution $Y$ is often dictated by the available statistics and by the experimental errors. The binning should not be chosen too fine in order to assure significant measurements in all bins. In the case of an IACT experiment, a sufficiently large sample is required to determine the number of signal (excess) events with sufficient accuracy. The binning should not be chosen too wide either, because the binning in $Y$ limits the reconstruction of the fine structure of the unfolded distribution $S$.

Another criterion for the binnings is the behavior of Gram's matrix $G$. A too fine binning for $S$ leads to strong correlations between neighboring columns of the migration matrix $M$, implying small eigenvalues of $G$, which lead to a large noise component of $S$. Given a certain choice of the binning in $E_{\text {est }}$ and thus of $n a$, the bin size in $E_{\text {true }}$ or $n b$ should be set such, that the system of linear equations (2) is not underconstrained. This usually leads to wider bins in $E_{\text {true }}$ than in $E_{\text {est }}$. In MAGIC a typical value of $\Delta \log _{10}\left(E_{\text {true }}\right) / \Delta \log _{10}\left(E_{\text {est }}\right)$ is 1.4 (see Section 10). 
It should also be noted that the unfolding procedure doesn't require equidistant bins, neither in $E_{\text {est }}$ nor in $E_{\text {true }}$.

\subsection{Completeness of the migration matrix}

If $n a 1 \leq i \leq n a 2$ specifies the range of bins of the measured distribution $Y$ which are to be considered in the unfolding procedure, also the range in $E_{\text {true }}$ to be considered in the unfolding has to be chosen properly: one has to make sure that all bins $j$ of $E_{\text {true }}$ are present, for which the column $j$ of the migration matrix $M_{i j}$ contributes to the selected bins of $Y$, i.e. for which at least one of the elements $M_{i j}(n a 1 \leq i \leq n a 2)$ is different from zero.

There is an exception to this rule, if for some reason certain bins $j$ of $E_{\text {true }}$ are not expected to contribute to the selected bins in $E_{\text {est }}$. This is for example the case if one of the factors $A_{x x x}^{k}$ in (22) is so small that $S_{k}$ can be neglected.

\subsection{Additional cuts}

As explained in Section 2.1, the distribution $Y$ and the migration matrix $M$ have to be produced under identical conditions (selections, cuts). If an additional cut is imposed when generating $Y$, also $M$ has to be recalculated before doing the unfolding. If this additional cut is a cut in $E_{\text {est }}$ one may proceed in the following way:

- Renormalize the columns $j$ of $M$ to the selected range in $E_{\text {est }}$ (see eq.(1)).

- Perform the unfolding of $Y$ in the usual way.

- Apply a correction $A_{\text {addcut }}^{j}$ to the unfolded distribution $S_{j}$, where $A_{\text {addcut }}^{j}$ is the renormalization factor for column $j$ of $M$.

\subsection{Starting values for the minimization}

In the cases where the unfolding procedure involves numerical minimizations, like the ones discussed in section 3.1, the minimization may not converge. This problem can be often solved by choosing different starting values. Another reason for non-convergence is discussed in Section 7.5. In MAGIC the standard choice of the starting distribution for $S$, and also of the prior distribution $\epsilon$ in Schmelling's method, is a distribution which is close to the measured distribution $Y$. 


\subsection{Components of $S$ which cannot be determined in the unfolding}

According to eq. (2) those $S_{j}$ for which the column $M_{i j}(i=1, \ldots n a)$ is a null vector have no influence on $Y$ and can therefore not be determined in the unfolding. These components should not be varied in the minimization because they would unnecessarily complicate the minimization process and may lead to non-convergence.

\subsection{Dependence on the assumptions made in the Monte Carlo simulation}

For the unfolding the migration matrix $M_{i j}$ is the crucial quantity. Obviously, if it doesn't describe the real migration of events correctly, the unfolding result will be wrong. This means that at fixed $j$, i.e. at fixed $E_{\text {true }}$, the MC simulation has to describe the migration in $E$ correctly. This will be the case if at fixed $E_{\text {true }}$ the shower simulation is realistic and if the detector response is simulated correctly.

On the other hand, the distribution of $E_{t r u e}$ in the MC need not agree with the real distribution of $E$ : due to the normalization of $M$ (eq. (1)) the bin-to-binvariation of the number of $\mathrm{MC}$ events in $E_{\text {true }}$ has no influence on $M_{i j}$ at all. This is one of the great advantages of unfolding methods like those presented in Section 3 as compared to methods based on correction factors (see Section $11)$.

However, there is a residual dependence of $M_{i j}$ due to the finite binning in $E_{\text {true }}$ : Depending on the shape of the $E_{\text {true }}$ distribution within an $E_{\text {true }}$ bin in the MC simulation, the calculated $M_{i j}$ may be more representative for the lower, middle or upper part of the $E_{\text {true }}$ bin. If the $E_{\text {true }}$ distribution in the real data is different from that in the $\mathrm{MC}$ simulation the calculated $M_{i j}$ may not be exactly the right ones.

This residual dependence of $M$ on the shape of the $E_{\text {true }}$ distribution in the $\mathrm{MC}$ can be nearly completely removed by an iteration procedure in which the $M$ for the next iteration step is determined from a MC sample, in which the $E_{\text {true }}$ distribution has been corrected using the unfolding result of the last iteration step (see Section 8). 


\section{Determining the effective collection area $A$ and the migration matrix $M$ for a finite bin in $E_{\text {true }}$ and $\Theta$}

In an IACT experiment it is important that the effective collection area $A$, which enters in the flux calculation, is computed taking into account a realistic shape of the differential flux $\Phi\left(E_{\text {true }}\right)$ and the actual distribution of effective observation times $d T(\Theta) / d \Theta$ in the zenith angle $\Theta$. This is also important for the migration matrix $M$, which enters in the unfolding, because of the residual dependence on the flux spectrum, as discussed in Section 7.6. Recalculating $A$ and $M$ with the proper $E_{\text {true }}$ and $\Theta$ spectra is the more important the bigger the $\left(\Delta E_{\text {true }}, \Delta \Theta\right)$ interval and the stronger the variations of $M$ and $A$ within this bin are. Often, for statistics reasons, large bin sizes in $E_{\text {est }}$ and $\Theta$, and thus also in $E_{\text {true }}$, have to be chosen.

In the following it is assumed that the effective collection area $A$ and the migration matrix $M$ are known functions of $E_{\text {true }}$ and $\Theta$. This can be achieved by determining them from a sample of MC $\gamma$-ray events in very fine bins of

$E_{\text {true }}$ and $\Theta$. The aim is to calculate an average $\bar{A}$ of the effective collection area and an average $\bar{M}$ of the migration matrix, which are representative for a finite bin $\left(\Delta E_{\text {true }}, \Delta \Theta\right)$ in $E_{\text {true }}$ and $\Theta$.

\subsection{The effective collection area}

The number of observed events in a $\left(\Delta E_{\text {true }}, \Delta \Theta\right)$ bin is given by

$$
S=\int_{\Delta \Theta} \int_{\Delta E_{\text {true }}} A\left(E_{\text {true }}, \Theta\right) \cdot \Phi\left(E_{\text {true }}\right) \cdot \frac{d T(\Theta)}{d \Theta} \cdot d E_{\text {true }} \cdot d \Theta
$$

Here $\Phi\left(E_{\text {true }}\right)$ is the differential $\gamma$-ray flux to be measured, $\frac{d T(\Theta)}{d \Theta}$ is the distribution of observation times in the experimental data and $A\left(E_{\text {true }}, \Theta\right)$ is the known dependence of the effective collection area on $E_{\text {true }}$ and $\Theta$.

With the definitions

$$
\begin{aligned}
\Delta T & \left.=\int_{\Delta \Theta} \frac{d T(\Theta)}{d \Theta} \cdot d \Theta \quad \text { (= total observation time }\right) \\
\bar{\Phi} & =\frac{1}{\Delta E_{\text {true }}} \int_{\Delta E_{\text {true }}} \Phi\left(E_{\text {true }}\right) \cdot d E_{\text {true }} \\
\bar{A} & =\frac{\int_{\Delta \Theta} \int_{\Delta E_{\text {true }}} A\left(E_{\text {true }}, \Theta\right) \cdot \Phi\left(E_{\text {true }}\right) \cdot \frac{d T(\Theta)}{d \Theta} \cdot d E_{\text {true }} \cdot d \Theta}{\Delta T \cdot \bar{\Phi} \cdot \Delta E_{\text {true }}}
\end{aligned}
$$


equation (25) can be rewritten as

$$
\bar{\Phi}=\frac{S}{\Delta T \cdot \bar{A} \cdot \Delta E_{\text {true }}}
$$

This is the usual formula for converting numbers of events $S$ into differential fluxes $\Phi$. Because of the definitions (27) and (28) the differential flux $\bar{\Phi}$ in an $E_{\text {true }}$ bin, as determined in the unfolding, is the average differential flux in this bin. Therefore, when quoting or plotting a result for $\bar{\Phi}$ the bin edges in $E_{\text {true }}$ should also be given or shown.

\subsection{The migration matrix}

The number of reconstructed $\mathrm{MC}$ events in a bin $i$ of $E_{\text {est }}$ can be written as

$$
\begin{aligned}
N_{i}= & \int_{\Delta \Theta} \int_{\Delta E_{\text {true }}} M_{i}\left(E_{\text {true }}, \Theta\right) \cdot A\left(E_{\text {true }}, \Theta\right) \cdot \Phi\left(E_{\text {true }}\right) \cdot \frac{d T(\Theta)}{d \Theta} \cdot d E_{\text {true }} \cdot d \Theta \\
& \text { with } \quad \sum_{k} M_{k}\left(E_{\text {true }}, \Theta\right)=1 \quad \text { for all } E_{\text {true }} \text { and } \Theta
\end{aligned}
$$

$M_{i}\left(E_{\text {true }}, \Theta\right)$ is the element of the normalized migration matrix for the $i$-th bin in $E_{\text {est }}$, at an energy $E_{\text {true }}$. The index $j$ of $M_{i j}$ is replaced by the variable $E_{\text {true }}$. The dependence of $M_{i}\left(E_{\text {true }}, \Theta\right)$ on $E_{\text {true }}$ and $\Theta$ is assumed to be known from MC simulations.

The average migration matrix $\bar{M}_{i}$ for the selected $\Delta E_{\text {true }}$ bin, to be used in the unfolding, is then obtained by

$$
\begin{aligned}
\bar{M}_{i} & =\frac{N_{i}}{\sum_{k} N_{k}} \\
& =\frac{\int_{\Delta \Theta} \int_{\Delta E_{\text {true }}} M_{i}\left(E_{\text {true }}, \Theta\right) \cdot A\left(E_{\text {true }}, \Theta\right) \cdot \Phi\left(E_{\text {true }}\right) \cdot \frac{d T(\Theta)}{d \Theta} \cdot d E_{\text {true }} \cdot d \Theta}{\int_{\Delta \Theta} \int_{\Delta E_{\text {true }}} A\left(E_{\text {true }}, \Theta\right) \cdot \Phi\left(E_{\text {true }}\right) \cdot \frac{d T(\Theta)}{d \Theta} \cdot d E_{\text {true }} \cdot d \Theta}
\end{aligned}
$$

The averages $\bar{M}_{i}$ and $\bar{A}$ are calculated according to (31) and (28) respectively, using an approximation $\Phi_{1}\left(E_{\text {true }}\right)$ of the function $\Phi\left(E_{\text {true }}\right)$. The measured distribution $Y$ is unfolded using $\bar{M}_{i}$, yielding the unfolded distribution $S$. A new approximation $\Phi_{2}\left(E_{\text {true }}\right)$ is then determined from $S$ according to eq. (29). The procedure is iterated until $\Phi\left(E_{\text {true }}\right)$ has converged. In practice parametric functions are used as approximations of $\Phi\left(E_{\text {true }}\right)$, and in general the convergence is found to be very fast. 


\section{Combining data before applying the unfolding procedure}

One often has the situation that there exist several measured distributions $Y^{\nu}$ of the same quantity. If the $Y^{\nu}$ were obtained under different conditions also the migration matrices $M^{\nu}$ and the effective collection areas $A^{\nu}$ will be different for the different measurements.

In an IACT experiment the different conditions may be

- Different modes of observation (ON/OFF mode, wobble mode, observation in the presence of moon light, ...).

- Different ranges of the zenith angle.

- Different detector conditions.

- etc.

In order to determine a final unfolded distribution $S$ one may proceed in different ways :

\section{- Individual unfolding :}

Unfold each $Y^{\nu}$ using $M^{\nu}$ to obtain $S^{\nu}$, and combine the $S^{\nu}$ to obtain the final solution $S$.

\section{- Global unfolding :}

Combine the $Y^{\nu}$ and $M^{\nu}$ to obtain a global $Y$ and $M$, do an unfolding of $Y$ using $M$, which will give the final solution $S$.

There is one important argument in favour of the second option : Each of the $Y^{\nu}$, or some of them, may have large statistical errors, making the unfolding of the individual $Y^{\nu}$ unstable. One common unfolding of the global $Y$ using the global $M$ will be more robust, in general.

In the case of an IACT experiment, the following relations hold for each measurement $\nu$ :

$$
\begin{aligned}
Y^{\nu} & =M^{\nu} \cdot S^{\nu} \\
S_{j}^{\nu} & =T^{\nu} \cdot A_{j}^{\nu} \cdot \Phi_{j} \cdot \Delta E_{j}
\end{aligned}
$$

$j$ denotes the $j$-th bin in $E_{\text {true }}, \Delta E_{j}$ is its width, $\Phi_{j}$ is the average flux and $A_{j}$ is the effective collection area in this bin, and $T$ is the effective observation time. Since $\Phi$ and $\Delta E$ are the same for all $\nu$ the relations for the combined data read :

$$
\begin{aligned}
Y & =M \cdot S \\
S_{j} & =T \cdot A_{j} \cdot \Phi_{j} \cdot \Delta E_{j}
\end{aligned}
$$


Inserting $Y=\sum_{\nu} Y^{\nu}$ and $T=\sum_{\nu} T^{\nu}$ in (33) and using (32) one obtains

$$
\begin{aligned}
A_{j} & =\frac{\sum_{\nu} A_{j}^{\nu} \cdot T^{\nu}}{T} \\
M_{i j} & =\frac{\sum_{\nu} M_{i j}^{\nu} \cdot\left(A_{j}^{\nu} T^{\nu}\right)}{\sum_{\mu}\left(A_{j}^{\mu} T^{\mu}\right)}=\frac{\sum_{\nu} M_{i j}^{\nu} \cdot\left(A_{j}^{\nu} T^{\nu}\right)}{A_{j} T}
\end{aligned}
$$

The relations (34) and (35) give the prescription how to combine the individual $M^{\nu}$ to obtain the global $M$, and how to combine the individual $A_{j}^{\nu}$ to obtain the global $A_{j}$. The measured distribution $Y$ is unfolded using $M$, and the unfolded distribution $S$ is converted into a flux $\Phi$ according to (33). As can be seen from (34) and (35) the $A_{j}$ are weighted averages of the $A_{j}^{\nu}$ with weights $w_{\nu}=T^{\nu} / T$, and the $M_{i j}$ are weighted averages of the $M_{i j}^{\nu}$ with weights $w_{\nu}=A_{j}^{\nu} T^{\nu} /\left(\sum_{\mu} A_{j}^{\mu} T^{\mu}\right)$. The weights can be interpreted as fractions because they add up to 1 . They are correlated and their covariance matrix has to be taken into account when calculating the errors of $A_{j}$ and $M_{i j}$.

The equations (34) and (35) also show that $A_{j}$ and $M_{i j}$ can be obtained without knowning which spectra $\Phi\left(E_{\text {true }}\right)$ and $d T(\Theta) / d \Theta$ were used to compute the $A_{j}^{\nu}$ and $M_{i j}^{\nu}$.

\section{Application to experimental data}

In this Section an experimental energy distribution of $\gamma$-rays from the Crab Nebula is unfolded, which was obtained in an analysis of data taken with the MAGIC telescope [24]. The migration matrix $M$, as determined from a sample of $\gamma$-MC events, is plotted in Fig. 2a) as a function of $E_{\text {est }}$ and $E_{\text {true }}$. The size of the boxes is proportional to the value of $M_{i j}$, where $i$ and $j$ are the bin numbers in $E_{\text {est }}$ and $E_{\text {true }}$ respectively. The experimental distribution $Y$ of the number of $\gamma$-excess events as a function of $E_{\text {est }}$ is displayed in Fig. 2b). Both distributions are after all cuts and selections, except a cut in $E_{\text {est }}$.

The vertical and horizonthal lines in Figs. 2a) and c) indicate the ranges in $E_{\text {est }}$ or $E_{\text {true }}$, which were selected for the unfolding. The range in $E_{\text {est }}$ is given by those $E_{\text {est }}$ bins for which a significant number of excess events could be determined. The range in $E_{\text {true }}$ comprises all those $E_{\text {true }}$ bins which are expected to contribute to the selected range in $E_{\text {est }}$. Fig. 2a) suggests that these are all $15 E_{\text {true }}$ bins. However, according to the plot of the effective collection area $A_{\text {eff }}$ in Fig. 2c) the contribution from $E_{\text {true }}<60 \mathrm{GeV}$ is expected to be negligible. The same holds for $E_{\text {true }}>9 \mathrm{TeV}$, due to the strongly decreasing $\gamma$-ray flux with increasing energy. This is confirmed by a Forward Unfolding of $Y$ in which a differential $\gamma$-ray flux of the form $\frac{d N}{d A \cdot d t \cdot d E}=f_{0} \cdot\left(\frac{E}{300 \mathrm{GeV}}\right)^{\alpha}$, 


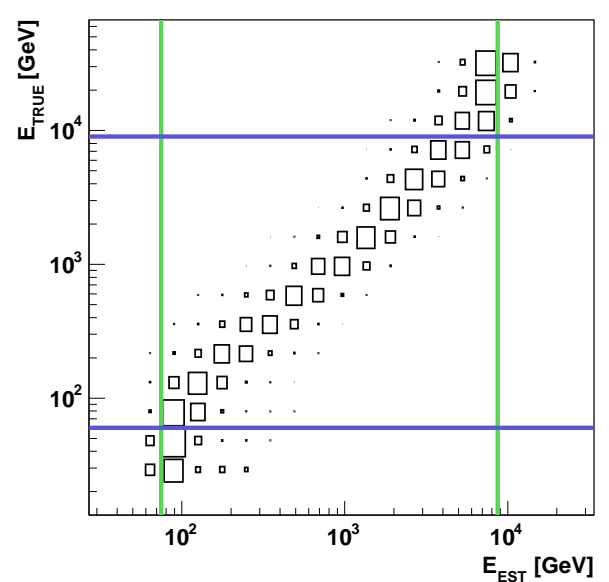

(a) Original migration matrix $M$

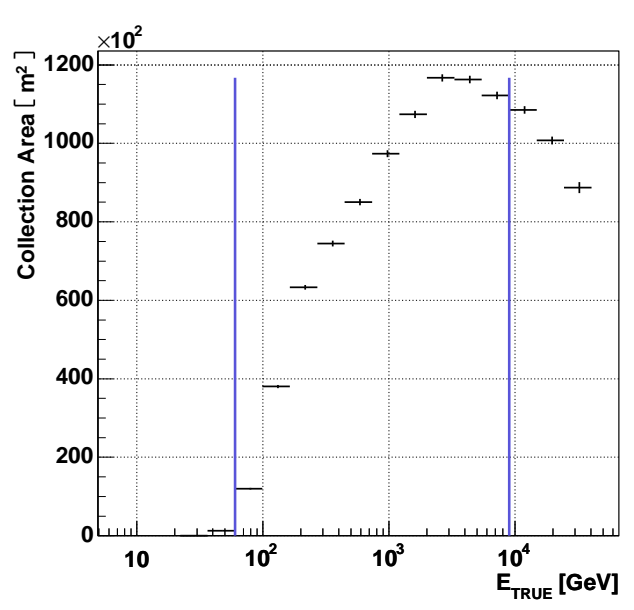

(c) Effective collection area $A_{\text {eff }}$

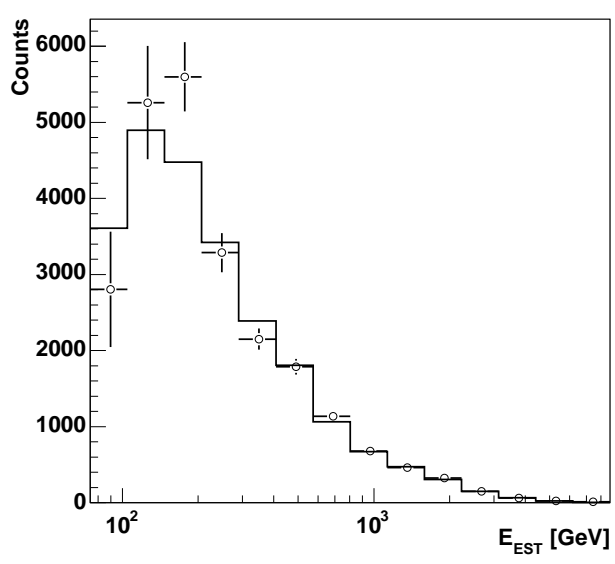

(b) Distribution $Y$ to be unfolded (open circles), and the unfolded distribution $S$ folded with $M$ (histogram)

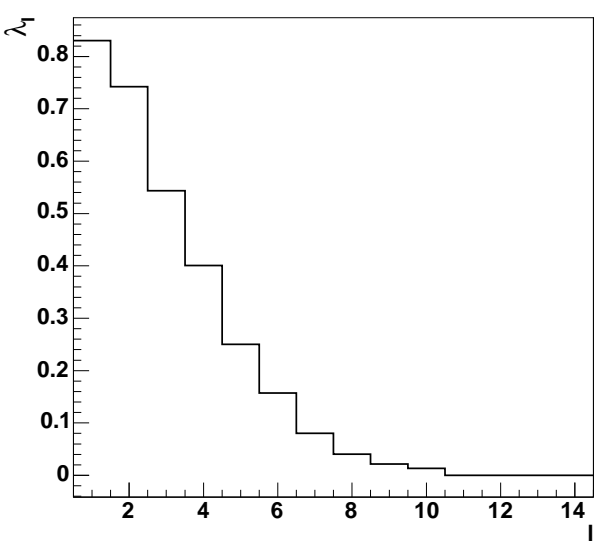

(d) Eigenvalues of Gram's matrix $G$

Fig. 2. Input data for the Unfolding and for the calculation of the differential $\gamma$-ray flux.

with $\alpha=a+b \cdot \log _{10}\left(\frac{E}{300 G e V}\right)$, was assumed. This leads to a number of $E_{\text {est }}$ and $E_{\text {true }}$ bins of $n a=14$ and $n b=10$ respectively, which are used in the unfolding procedure. The size of the $\log _{10}\left(E_{\text {true }}\right)$ bins was deliberately chosen wider than the $\log _{10}\left(E_{\text {est }}\right)$ bins by a factor of 1.4 , in order to better constrain the unfolding. The rank of Gram's matrix $G$ is equal to $n r=10$, as can be seen from Fig. 2d), which shows the size of the eigenvalues $\lambda_{l}$ of $G$ as a function of $l$. Two to three of these eigenvalues are much smaller than the maximum eigenvalue, and they are the reason for the large values of $\operatorname{Trace}(T) / \operatorname{Trace}(K)$ at large iteration number (low regularization strength) in Fig. 1b).

The optimum regularization strength and thus the final solution $S$ was determined using the criterion $\operatorname{Trace}(T) / \operatorname{Trace}(K)=1$. The estimates $\sum_{j} M_{i j} \cdot S_{j}$ 

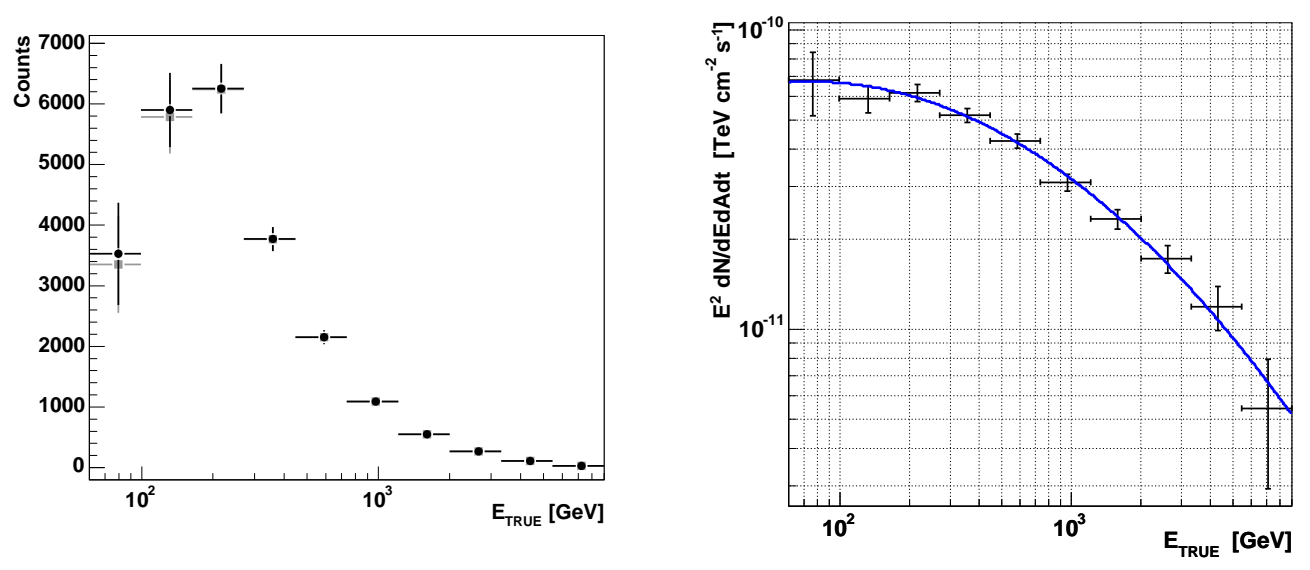

(a) Unfolded distribution $S$ before (grey (b) Result for the differential $\gamma$-ray flux symbols) and after the correction for the $\Phi\left(E_{\text {true }}\right)$ multiplied with $E^{2}$. cut in $E_{\text {est }}$ (black symbols).

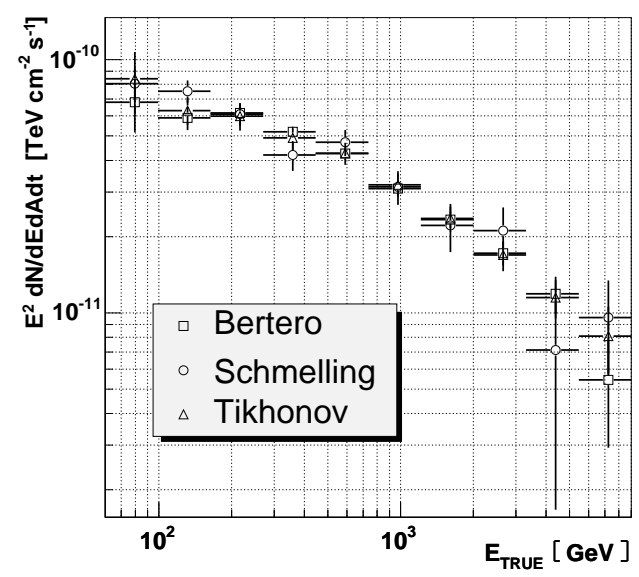

(c) Comparison of the results for $E^{2}$. $\Phi\left(E_{\text {true }}\right)$ from different unfolding methods.

Fig. 3. Results from the Unfolding.

(open circles) of $Y_{i}$ are compared with $Y_{i}$ (histogram) in Fig. 2b). These data enter in the calculation of $\chi_{0}^{2}$ (eq. 3). The number of degrees of freedom in the unfolding procedure is $n a-n b+1=5$, because the number of measurements is $n a$, the number of unknowns is $n b$, and the relation $\sum_{j} S_{j}=\sum_{i} Y_{i}$ (eq. 6) is used as additional constraint. As can be seen from Figs. 1d) and e) the values of $\operatorname{Reg}(S)_{\text {Tikhonov }}$ and $\operatorname{Reg}(S)_{\text {Schmelling }}$ are much lower at the selected regularization strength than without regularization. This means that the solution $S$ is smoothed by the regularization. The black symbols in Fig. 3a) represent the final solution $S$. The solution before correctiong for the cut in $E_{\text {est }}$ is drawn with grey symbols. 
The final differential $\gamma$-ray flux $\Phi$, as computed from $S$ according to eq.(22), is drawn in Fig. 3b). The solid line represents the result of a fit of the expression $f_{0} \cdot\left(\frac{E}{300 G e V}\right)^{\alpha}$, with $\alpha=a+b \cdot \log _{10}\left(\frac{E}{300 G e V}\right)$, to the data points. The number of degrees of freedom for this fit is $n b-3=7$, because the number of data points is $n b=10$ and the number of free parameters is $3\left(f_{0}, a\right.$ and $b)$. The $\chi^{2}$ is 8 for 7 degrees of freedom. Setting $b=0$ in the fit yields a $\chi^{2}$ of 24 for 8 degrees of freedom. This fit is clearly disfavoured as compared to the fit in which the slope $\alpha$ is energy dependent. In these fits the full correlation matrix $T$ of $S$ has been taken into account.

The result of the latter fit for $\Phi\left(E_{\text {true }}\right)$ was used to recalculate the averages $\bar{M}_{i}$ (31) and $\bar{A}$ (28) for the individual $E_{\text {true }}$ bins. The unfolding was repeated using the recalculated $\bar{M}_{i}$ and $\bar{A}$, yielding new results for $S, \Phi$ and the fit parameters. After 1 iteration this procedure converged.

Very similar results were obtained with the other unfolding methods. The spread of the $S_{j}$, obtained with the different unfolding methods, can be seen in Fig. 3c). This spread can be regarded as an estimate of the a systematic error due to the unfolding.

\section{Correction Factors}

A widely used method of correcting experimental distributions is the application of correction factors: Using Monte Carlo data, both the 'true' distribution $S_{k}^{M C} \quad(k=1, \ldots n c)$ and the 'reconstructed' distribution $Y_{k}^{M C} \quad(k=1, \ldots n c)$ of some quantity are produced under certain conditions (selections, cuts). Correction factors are determined according to

$$
c_{k}=S_{k}^{M C} / Y_{k}^{M C} \quad(k=1, \ldots n c)
$$

An experimental distribution $Y_{k}$, obtained under the same conditions as $Y_{k}^{M C}$, is then corrected by

$$
S_{k}=Y_{k} \cdot c_{k} \quad(k=1, \ldots n c)
$$

to obtain the corrected distribution $S_{k}$.

The following properties of this procedure can be stated [17] :

- $c_{k}$ is undefined if $Y_{k}^{M C}=0$.

- If $S_{k}^{M C}=0$ also $c_{k}=0$ and $S_{k}=0$, which means that $Y_{k}$ is ignored. 
- $c_{k}$ depends on the shape of the MC distribution $S_{k}^{M C}$; the corrected distribution $S_{k}$ is always biased towards $S_{k}^{M C}$.

- If $Y_{k}=0$ also $S_{k}$ is zero.

- The standard linear error propagation often yields too small errors of $S_{k}$.

The correction factors are only right if $S_{k}^{M C}$ is identical to the true $S_{k}$ distribution. If this is not the case one may iterate $S_{k}^{M C}$, setting $S_{k}^{M C}$ equal to the last corrected experimental distribution $S_{k}$. However, this often leads to instabilities. The reason for the instabilities appears to be similar to that causing a large noise component of the direct solution (4).

In contrast to the unfolding methods presented in Section 3, there is very little freedom in choosing the binnings for $S$ and $Y$. By definition, the range of values and the binnings for the true and reconstructed quantity are identical.

Advantages of the method of correction factors are that it is simple and stable. The drawbacks have been listed above, the severest one being the strong dependence of the correction factors on the assumptions made in the MC about $S$.

\section{Summary}

In this paper the procedures to unfold experimental energy distributions of $\gamma$ rays, as applied in the MAGIC experiment, are described. It is explained, how the uncertainties, which are inherent in any unfolding process, can be handled successfully. Possible problems in the unfolding are discussed and suggestions are given which can help to avoid them. Various techniques are presented, which allow to reconstruct the energy spectrum in a rather unbiased way. All algorithms are impleneted in the MAGIC software, which is based on the $\mathrm{C}^{++}$ language and ROOT [25]. Their application to real data has shown to provide robust and reliable results. The methods and procedures are applied in most of the MAGIC analyses.

\section{A Acknowledgements}

We thank Michael Schmelling for fruitful discussions and critical comments. 


\section{References}

[1] Baixeras, C. et al., 2004, Nucl. Instrum. Meth., A518, 188.

[2] R. Gold, ANL-6984 (1964)

[3] G.I. Marchuk, "Methods of Numerical Mathematics", Springer, Berlin (1975)

[4] A.N. Tikhonov and V.Ja. Arsenin, Methods of Solution of Ill-posed Problems M (Nauka, 1979)

[5] S.W. Provencher, Computer Physics Communications 27 (1982) 213 and 229

[6] V. Blobel, "Unfolding methods in high-energy physics experiments", DESY 84118 (1984).

[7] V. Blobel, 1984 CERN School of Computing, Ajguablava, Spain, CERN 85-09 (1984) p.88.

[8] E.A. Belogorlov et al., "Interpretation of the solution to the inverse problem for the positive function and the reconstruction of neutron spectra", NIM A 235 (1985) 146.

[9] S.F. Giljazov, "Methods of Solution of Linear Ill-posed Problems", MSU, Moscow (1987)

[10] V.P. Zhigunov et al., "On estimating distributions with the maximum entropy principle", NIM A 273 (1988) 362

[11] M. Bertero, INFN/TC-88/2 (1988).

[12] M. Bertero, Advances in Electronics and Electron Physics, Vol.75 (1989).

[13] V.B. Anykeyev et al., NIM A 303 (1991) 350.

[14] M. Schmelling, "The method of reduced cross-entropy. A general approach to unfold probability distributions", NIM A 340 (1994) 400.

[15] V. Blobel, "The RUN manual", OPAL Technical Note TN361 (1996)

[16] A. Höcker and V. Kartvelishvili, NIM A 372 (1996) 469.

[17] M. Schmelling, "Numerische Methoden der Datenanalyse", MPI-K Heidelberg (1998)

[18] V. Blobel, "An Unfolding Method for High Energy Physics", IPPP Workshop on Advanced Statistics Techniques in Particle Physics, Durham (2002)

[19] W. Wittek," Correlations between Parameters of Extended Air Showers and their Proper Use in Analyses", 26th Int. Cosmic Ray Conference, Salt Lake City, Utah, USA (1999)

[20] Majumdar, P. et al. (MAGIC Collab.), 2005, Proc. of the 29th ICRC, Pune, India, 5-203, astro-ph/0508274. 
[21] Mizobuchi, S. et al. (MAGIC Collab.), Proc. 29th ICRC, Pune, India, 5-323, astro-ph/0508274.

[22] Bretz, T. and Wagner, R. (MAGIC Collab.), 2003, Proceedings of the $28^{\text {th }}$ ICRC, Tsukuba, Japan, 2947.

[23] T.M. Kneiske et al., A\&A 413 (2004) 807

[24] J. Albert et al., submitted for publication in ApJ (2007), arXiv:0705.3244.

[25] R. Brun, F. Rademakers, http://root.cern.ch/. 